\title{
Adipose tissue hypoxia induces inflammatory M1 polarity of macrophages in an HIF- $1 \alpha$-dependent and HIF- $1 \alpha$-independent manner in obese mice
}

\author{
S. Fujisaka • I. Usui • M. Ikutani - A. Aminuddin • \\ A. Takikawa $\cdot$ K. Tsuneyama $\cdot$ A. Mahmood $\cdot$ N. Goda • \\ Y. Nagai $\cdot$ K. Takatsu $\cdot$ K. Tobe
}

Received: 20 November 2012 / Accepted: 26 February 2013 / Published online: 15 March 2013

(C) Springer-Verlag Berlin Heidelberg 2013

\begin{abstract}
Aims/hypothesis As obesity progresses, adipose tissue exhibits a hypoxic and inflammatory phenotype characterised by the infiltration of adipose tissue macrophages (ATMs). In this study, we examined how adipose tissue hypoxia is involved in the induction of the inflammatory M1 and anti-inflammatory M2 polarities of ATMs.

Methods The hypoxic characteristics of ATMs were evaluated using flow cytometry after the injection of pimonidazole, a hypoxia probe, in normal-chow-fed or high-fat-fed mice. The
\end{abstract}

Electronic supplementary material The online version of this article (doi:10.1007/s00125-013-2885-1) contains peer-reviewed but unedited supplementary material, which is available to authorised users.

S. Fujisaka $\cdot$ I. Usui $(\bowtie) \cdot$ A. Aminuddin $\cdot$ A. Takikawa

A. Mahmood $\cdot K$. Tobe $(\bowtie)$

First Department of Internal Medicine, University of Toyama,

2630 Sugitani, Toyama 930-0194, Japan

e-mail: isaousui-tym@umin.ac.jp

e-mail: tobe-tky@umin.ac.jp

M. Ikutani $\cdot$ Y. Nagai $\cdot$ K. Takatsu

Department of Immunobiology and Pharmacological Genetics, Graduate School of Medicine and Pharmaceutical Science

for Research, University of Toyama, Toyama, Japan

K. Tsuneyama

Department of Diagnostic Pathology, University of Toyama,

Toyama, Japan

N. Goda

Department of Life Science and Medical Bioscience, School of Advanced Science and Engineering, Waseda University, Tokyo, Japan

K. Takatsu

Toyama Prefectural Institute for Pharmaceutical Research,

Toyama, Japan expression of hypoxia-related and inflammation-related genes was then examined in M1/M2 ATMs and cultured macrophages. Results Pimonidazole uptake was greater in M1 ATMs than in M2 ATMs. This uptake was paralleled by the levels of inflammatory cytokines, such as TNF- $\alpha$, IL- 6 and IL- $1 \beta$. The expression level of hypoxia-related genes, as well as inflammation-related genes, was also higher in M1 ATMs than in M2 ATMs. The expression of $I l 6, I l 1 \beta$ and Nos 2 in cultured macrophages was increased by exposure to hypoxia in vitro but was markedly decreased by the gene deletion of Hifla. In contrast, the expression of Tnf, another inflammatory cytokine gene, was neither increased by exposure to hypoxia nor affected by Hifla deficiency. These results suggest that hypoxia induces the inflammatory phenotypes of macrophages via Hifla-dependent and -independent mechanisms. On the other hand, the expression of inflammatory genes in cultured M2 macrophages treated with IL-4 responded poorly to hypoxia.

Conclusions/interpretation Adipose tissue hypoxia induces an inflammatory phenotype via Hifla-dependent and Hiflaindependent mechanisms in M1 ATMs but not in M2 ATMs.

Keywords Adipose tissue macrophage $\cdot$ HIF- $1 \alpha \cdot$ Hypoxia $\cdot$ Inflammatory cytokine $\cdot \mathrm{M} 1$ macrophage

$\begin{array}{ll}\text { Abbreviations } \\ \text { APC } & \text { Allophycocyanin } \\ \text { ATM } & \text { Adipose tissue macrophage } \\ \text { BMDM } & \text { Bone-marrow-derived macrophage } \\ \text { CD } & \text { Cluster of differentiation } \\ \text { CLS } & \text { Crown-like structure } \\ \text { Cy7 } & \text { Carbocyanin7 } \\ \text { HFD } & \text { High-fat diet } \\ \text { HIF } & \text { Hypoxia-inducible factor }\end{array}$




$\begin{array}{ll}\text { LysM } & \text { Lysozyme M } \\ \text { M-CSF } & \text { Macrophage colony-stimulating factor } \\ \text { NF-kB } & \text { Nuclear factor-kappaB } \\ \text { PE } & \text { Phycoerythrin } \\ \text { SVF } & \text { Stromal vascular fraction } \\ \text { VEGF } & \text { Vascular endothelial growth factor }\end{array}$

\section{Introduction}

Chronic and low-grade inflammation is a pivotal pathogenetic mechanism of various obesity-associated diseases including the metabolic syndrome and type 2 diabetes [1-3]. Visceral adipose tissue is a major site for such chronic inflammatory responses and adipose tissue macrophages (ATMs) perform important roles, such as the production of inflammatory cytokines [2-4], therein. In general, macrophages exhibit marked functional heterogeneity and local microenvironments influence their activation status and functions $[5,6]$. Th1 cytokines, such as IFN- $\gamma$, induce classical activation of M1 macrophages, which produce inflammatory mediators. Th2 cytokines, such as IL-4 and IL-13, induce the alternative activation of M2 macrophages, which mediate anti-inflammatory responses. M1 ATMs produce inflammatory cytokines, such as TNF- $\alpha$ and IL-6, that are involved in the induction of insulin resistance. The majority of ATMs in lean individuals, however, exhibit an anti-inflammatory M2 polarity. These ATMs reportedly play several roles in the maintenance or improvement of insulin sensitivity [7-12]. In our recent study, flow-cytometry analysis using cluster of differentiation (CD)11c and CD206 as M1 and M2 ATM markers, respectively, revealed that the number of M1 ATMs increased dramatically in mice fed a high-fat diet (HFD), while the increase in the number of M2 ATMs was modest [7].

In a recent review Sun et al stated that at least four factors may trigger an increase in the number of M1 ATMs in visceral adipose tissue according to the progress of obesity — adipocyte death, chemotactic regulation, fatty-acid flux and adiposetissue hypoxia [13]. The oxygen partial pressure in adipose tissues is reported to decrease with obesity in both humans and rodents [14-20]. Several studies have suggested that hypoxia is involved in the remodelling process, which includes inflammatory and fibrotic changes in obese adipose tissues $[14,15$, $21,22]$. While hypoxia in adipocytes is involved in the fibrotic remodelling of adipose tissue, hypoxia in macrophages has been implicated in inflammatory changes. However, exactly how, and by what mechanisms, hypoxia affects the characteristics of M1 and M2 ATMs in adipose tissue from obese mice and leads to inflammation has not been fully addressed to date.

Subcutaneous adipose tissue is reported to exhibit less inflammation than visceral adipose tissue even in obese humans and rodents [23-26]. The oxygen partial pressure is reported to be decreased not only in visceral adipose tissues but also in subcutaneous adipose tissue [27]. How adipose tissue hypoxia exerts different effects on inflammatory changes in subcutaneous and visceral adipose tissues via M1/M2 ATMs remains elusive.

Here, we demonstrate the relationship between adipose tissue hypoxia and the inflammatory polarity of ATMs in high-fat-fed obese mice. We found that ATMs expressing a higher content of marker genes for hypoxia were more inflammatory and that M1 ATMs expressed hypoxiarelated genes more robustly than M2 ATMs in epididymal adipose tissue. When cultured macrophages were subjected to hypoxia in vitro, the expression of some M1 marker genes was upregulated in a hypoxia-inducible factor- $1 \alpha$ (HIF- $1 \alpha)$ dependent manner. In addition, exposure to hypoxia induced expression of some of the inflammatory genes to a lesser extent in IL-4-treated macrophages. From these experiments, we demonstrate that adipose tissue hypoxia induces M1-like polarity in ATMs, thus contributing to the inflammatory characteristics of visceral adipose tissue.

\section{Methods}

Materials Rat F4/80 antibody conjugated with allophy cocyanin (APC)/carbocyanin7 (Cy7), hamster CD11c antibody conjugated with phycoerythrin and rabbit $\beta$-actin antibody were purchased from BioLegend (San Diego, CA, USA), BD Biosciences (San Jose, CA, USA) and Cell Signaling (Danvers, MA, USA), respectively. Rat F4/80 antibody and rat CD206 antibody conjugated with APC were purchased from AbD serotec (Oxford, UK). Mouse CD45 antibody conjugated with phycoerythrin (PE)-Cy7, rat IL- 6 antibody conjugated with PE and rat TNF- $\alpha$ antibody conjugated with $\mathrm{PE}$ were purchased from eBioscience (San Diego, CA, USA). Biotinylated IL-1 $\beta$ antibody and streptavidin conjugated with APC were purchased from Novus Biologicals (Littleton, CO, USA) and BD Biosciences (San Jose, CA, USA), respectively. Mouse antibodies to HIF-1 $\alpha$ were purchased from Gene Tex (Irvine, CA, USA) and R\&D Systems (Minneapolis, MN, USA).

Maintenance of mice Six-week-old male C57BL/6J mice were purchased from CLEA Japan (Tokyo, Japan). The Hifl $a^{\text {flox/flox }} /$ LysM-cre knockout mice were generated by crossing a C57BL/6J mouse containing loxP sequences on either side of the Hifla gene with a C57BL/6J mouse expressing cre recombinase from the lysozyme M (LysM) promoter, which is found only in myeloid lineage cells. As a result, a homozygous mouse that was deficient in Hifla in monocytes and macrophages was generated. Mice containing the floxed Hifla allele, which does not express the cre recombinase gene ( $\left.H i f l a^{\text {flox/flox }}\right)$, served as the control in this 
study. The mice were maintained under a standard light cycle (12 h light/dark) and were allowed free access to water and food. They were fed a standard diet (chow) containing 10\% energy from fat (Nosan Corporation, Yokohama, Japan) or an HFD containing $60 \%$ fat (Research Diets, NJ, USA). The animal care policies and procedures for the experiments were approved by the animal experiment committee at the University of Toyama.

Hypoxia probe Flow cytometry, western blotting and immunohistostaining for the hypoxia probe were performed using the Hypoxyprobe-1 plus kit (hpi, Burlington, MA, USA) according to the manufacturer's instructions. Pimonidazole was intraperitoneally injected $30 \mathrm{~min}$ before tissue collection at a dose of $60 \mathrm{mg} / \mathrm{kg}$ body weight.

Flow cytometry analysis Flow cytometry of the stromal vascular fractions (SVFs) was performed as previously described $[7,8]$. M1 or M2 macrophages were identified as CD45positive/F4/80-positive/CD11c-positive/CD206-negative or CD45 positive/F4/80-positive/CD11c-negative/CD206-positive cells, respectively. For the pimonidazole, TNF- $\alpha$, IL-6 and IL-1 $\beta$ analyses, intracellular staining was conducted using IntraPrep (Beckman Coulter, CA, USA) according to the manufacturer's instructions. Briefly, SVFs incubated with antibodies for CD45, F4/80, 7AAD and/or CD11c, CD206 were fixed, permeability was induced and the fractions were incubated with antibodies for pimonidazole, TNF- $\alpha$, IL-6 or IL-1 $\beta$.

Isolation of adipocytes, SVFs and bone marrow-derived macrophages Adipocytes and SVFs were isolated as previously described [7, 8]. To isolate bone-marrow-derived macrophages (BMDMs), bone marrow was collected from femurs and tibias of 8- to 12-week-old mice. Cells were cultured in RPMI1640 supplemented with 10\% heat-inactivated FBS, $100 \mathrm{U} / \mathrm{ml}$ penicillin, $100 \mu \mathrm{g} / \mathrm{ml}$ streptomycin, $55 \mu \mathrm{mol} / 1 \beta$ mercaptoethanol and $100 \mathrm{ng} / \mathrm{ml}$ recombinant murine macrophage colony-stimulating factor (M-CSF; R\&D systems) for 7 days. For alternative macrophage activation, macrophages were stimulated with $10 \mathrm{ng} / \mathrm{ml}$ murine IL-4 (PeproTech, Rocky Hill, NJ, USA) for $24 \mathrm{~h}$. Differentiated BMDMs or SVFs were serum-starved and cultured under normoxic $(20 \%$ $\left.\mathrm{O}_{2}, 5 \% \mathrm{CO}_{2}\right)$ or hypoxic $\left(1 \% \mathrm{O}_{2}, 5 \% \mathrm{CO}_{2}\right)$ conditions for $24 \mathrm{~h}$.

Quantitative RT-PCR and western blot Fresh adipose tissue was frozen immediately in liquid nitrogen after isolation until being used in the experiments. Extraction of total RNA and quantitative RT-PCR were performed as previously described [7, 8]. Western blot analysis was performed as described previously [14] with a slight modification (i.e. sonication was needed for the preparation of cell lysate only for HIF-1 $\alpha$ blotting).
Immunohistochemistry of adipose tissues Immunohisto staining for F4/80 (rat monoclonal antibody; AbD serotec) and HIF-1 $\alpha$ (mouse monoclonal antibody; GeneTex) was performed as previously described [7]. The fresh adipose tissue was fixed in 4\% paraformaldehyde, dehydrated and embedded in paraffin. For HIF-1 $\alpha$ staining, a mouse-onmouse immunodetection kit (M.O.M.; Vector Laboratories, Burlingame, CA, USA) was used for special blocking. After incubation with the primary antibody, Histofine-peroxidase kit for rat (Nichirei, Tokyo, Japan) or an Envisionperoxidase kit for mouse (DAKO, Carpinteria, CA, USA) was applied as a secondary antibody. 3,3'-Diaminobenzidine was used as a substrate of peroxidase. Samples stained by F4/80 were then incubated with alkaline-phosphataseconjugated antibody against pimonidazole. Fast red (Vector Laboratories), which is visible under both light microscope and fluorescent microscope was used as a substrate of alkaline phosphatase.

Statistical analysis Statistical significance was evaluated using an ANOVA and a post Tukey-Kramer test or unpaired $t$ test. Differences were considered statistically significant at $p<0.05$. The results were presented as the means \pm SEM.

\section{Results}

Hypoxia-related genes were upregulated mainly in the SVF of adipose tissue in obese mice We first evaluated the levels of hypoxia in adipose tissues using pimonidazole hydrochloride, a chemical probe for hypoxia, and the content of HIF1- $\alpha$, a well-known transcription factor that can be activated by hypoxia. As shown in Fig. 1a, the pimonidazole signal and HIF- $1 \alpha$ content were increased in the epididymal adipose tissue of mice fed an HFD for 12 weeks. We next examined which compartment of adipose tissues was hypoxic during obesity. Epididymal adipose tissue was separated into two fractions - an SVF and an adipocyte fraction. The increase in expression of hypoxia-related genes was significantly greater in the SVF than in the adipocyte fraction of high-fat-fed mice (Fig. 1b-j). These data suggest that the adipose tissue of obese mice is characterised by hypoxia and that the SVF, including ATMs, may be the major site for the hypoxic response.

M1 ATMs become more hypoxic than M2 ATMs after highfat feeding We next examined whether ATMs became hypoxic during obesity. First, the number of cells positive for F4/80, a marker for mature macrophages, in the SVF was analysed using flow cytometry after pimonidazole injection. The number of ATMs with stronger pimonidazole signals was markedly increased in epididymal adipose tissue of high-fat-fed mice, compared with chow-fed mice (Fig. 2a). 

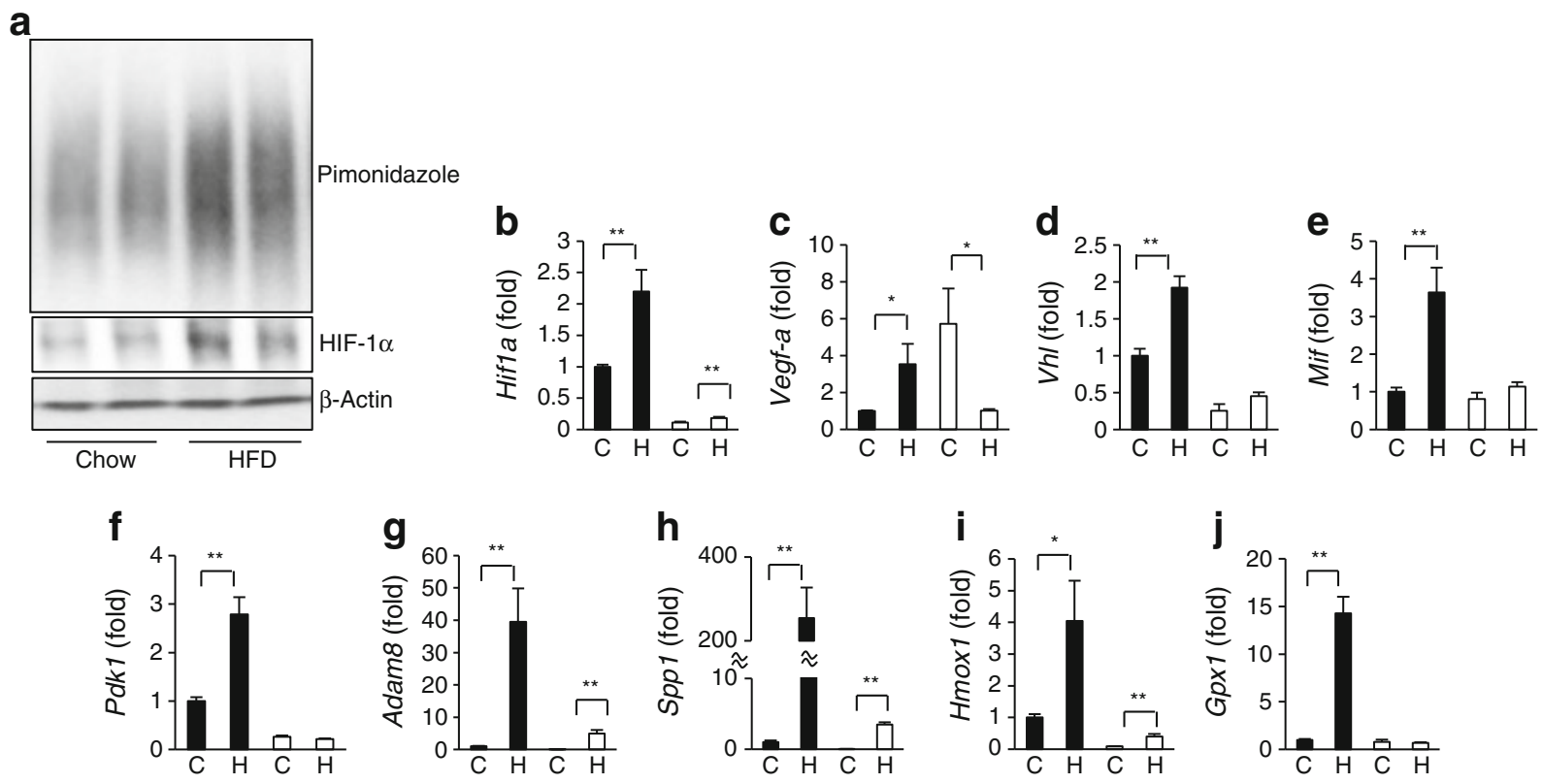

Fig. 1 Hypoxia-related markers were upregulated in adipose tissues of obese mice. (a) Representative western blot images of epididymal adipose tissue from 12 week chow-fed (chow) and high-fat-fed (HFD) C57BL/6J mice with anti-pimonidazole, anti-HIF-1 $\alpha$, and anti- $\beta$-actin antibodies. (b-j) Gene expression in the SVF (black bar) and

Second, the pimonidazole signals in the M1 and M2 ATMs were separately evaluated and compared. After high-fat feeding, the pimonidazole signals were enhanced in both the M1 and M2 ATMs. However, the intensity of the signals in the M1 ATMs became remarkably stronger than that of the signals in M2 ATMs in epididymal adipose tissues (Fig. 2b-e) and subcutaneous adipose tissues (Fig. 2f-i) at 8 and 12 weeks, respectively, after the start of high-fat feeding. The immunostaining of epididymal adipose tissues from high-fat-fed mice revealed that the pimonidazole signal was mainly detected around F4/80-positive crown-like structures (CLS), which are known to consist of M1 ATMs (Fig. $2 \mathrm{k}, 1)$. HIF-1 $\alpha$ staining was also strongly detected around the CLS (Fig. $2 \mathrm{~m}$ ). In contrast, pimonidazole or HIF- $1 \alpha$ signal in epididymal fat was much weaker in chow-fed mice, probably because almost no CLS was observed in these mice (Fig. $2 n-q$ ). These results suggest that M1 ATMs mainly become hypoxic in adipose tissue according to the progress of obesity.

In agreement with a recent report [7], the number of M1 ATMs was dramatically increased in the epididymal adipose tissues of mice fed an HFD for 12 weeks (Fig. 2r,s). In contrast, the number of M1 ATMs was relatively small and was not altered in the subcutaneous adipose tissues even after high-fat feeding (Fig. 2t,u), although M1 ATMs were clearly hypoxic (Fig. $2 \mathrm{f}-\mathrm{i}$ ). These results suggest that adipose tissue hypoxia alone is not sufficient to explain the increase in the number of M1 ATMs, at least in subcutaneous adipose tissues. adipocyte fraction (white bar) from chow-fed mice (C) or high-fat-fed mice $(\mathrm{H})$. Each data point was normalised according to the $18 \mathrm{~S}$ rRNA level and presented as a value relative to that for age-matched control mice. The results are shown as the mean \pm SEM for three or four mice per group. ${ }^{*} p<0.05, * * p<0.01$

ATMs expressing hypoxia marker genes also produce inflammatory cytokines The relationship between hypoxia and inflammation in ATMs was investigated. First, the gene profiles of M1 and M2 ATMs obtained from mice fed an HFD for 12 weeks were examined (Fig. 3a). As we previously reported [7], M1 ATMs highly expressed M1 marker genes, including those for inflammatory cytokines, such as Tnf, Il6 and Illb whereas M2 ATMs expressed clearly different gene profiles. The hypoxia-related genes, such as Hifla, Vegfa, Glut1 (also known as Slc2a1), Pdk1, Gpx1, $\mathrm{Vhl}$ and Adam8, were significantly elevated in M1 ATMs as well as other M1 marker genes (Fig. 3a). Then, the time courses of some genes related to hypoxia and inflammation were compared after high-fat feeding (see electronic supplementary material [ESM] Fig. 1). Most of the hypoxia-related genes, such as Vegfa, Glutl, Gpxl and Vhl, were significantly upregulated after 4 weeks of high-fat feeding in M1 ATMs. In contrast, the changes in the gene expression of M2 ATMs were smaller than those in the M1 ATMs (ESM Fig. 1a-e). Similarly, the expression of some inflammatory genes, such as Tnf and $I l l b$, was also increased after 4 weeks of high-fat feeding in M1 ATMs (ESM Fig. 1f,g). These results suggest that a close relationship exists between hypoxia and inflammatory responses in M1 ATMs after high-fat feeding. The relationship between hypoxia and inflammation in ATMs was also evaluated at the protein level using flow cytometry of ATMs after the injection of pimonidazole in mice. 

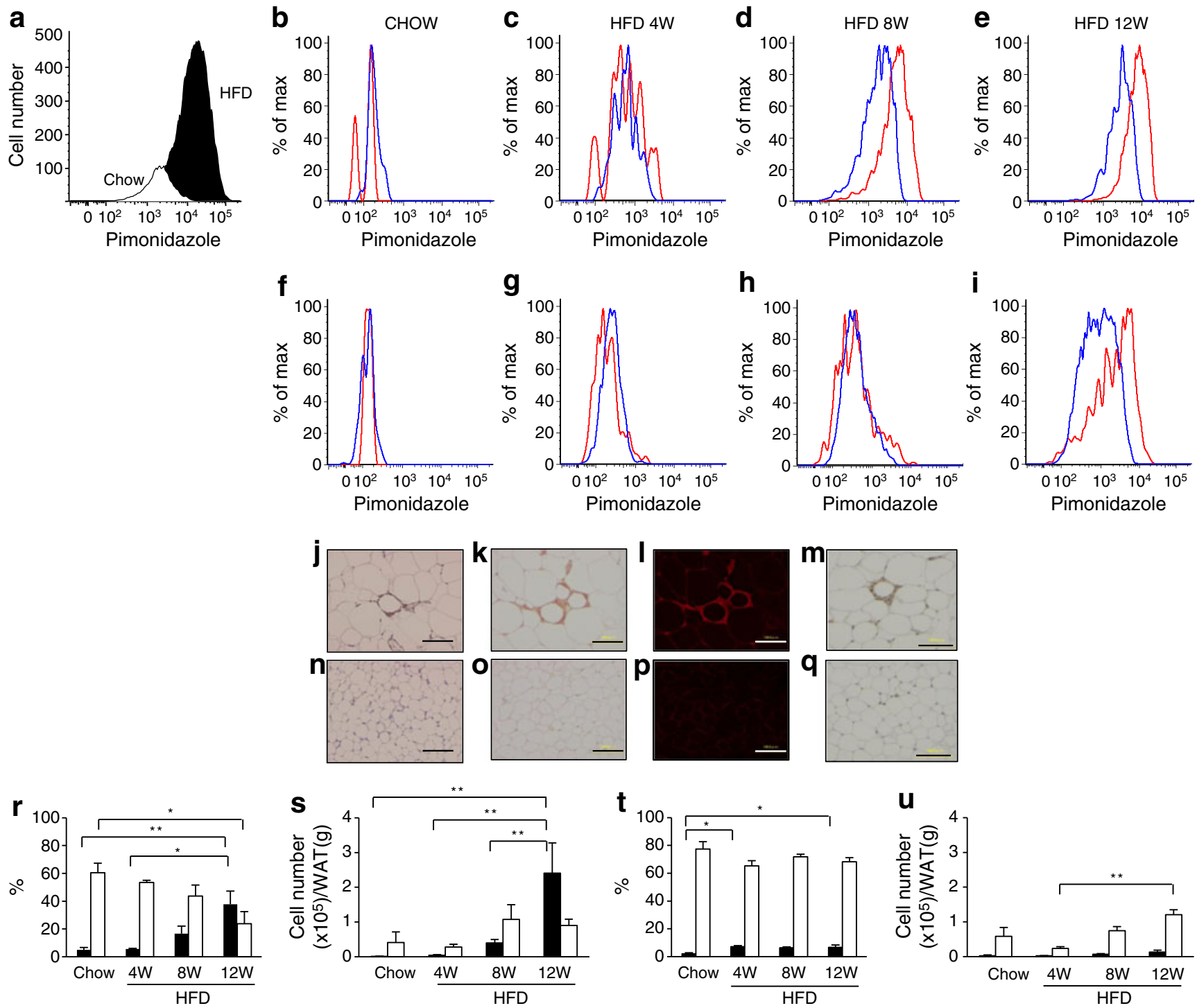

Fig. 2 M1 ATMs become more hypoxic than M2 ATMs after high-fat feeding. (a) Flow cytometry analysis of pimonidazole signals on CD45-positive, F4/80-positive cells in the SVF of epididymal adipose tissue from 12 week (W) chow-fed (chow) and high-fat-fed (HFD) C57BL/6J mice. (b-i) Pimonidazole signals in M1 (red lines) and M2 (blue lines) ATMs from epididymal adipose tissue (b-e) or subcutaneous adipose tissue (f-i) of chow-fed mice or mice fed an HFD for 4 , 8 or 12 weeks. All data are representative of at least four independent experiments. ( $\mathbf{j}-\mathbf{q})$ Immunostaining of epididymal adipose tissues from high-fat-fed $(\mathbf{j}-\mathbf{m})$ and normal chow-fed $(\mathbf{n}-\mathbf{q})$ mice. Epididymal

As shown in Fig. 3b, the ATMs were separated into two fractions according to the levels of pimonidazole staining. ATMs with strong pimonidazole staining produced higher levels of inflammatory cytokines, such as TNF $\alpha$, IL-6, and IL-1 $\beta$, than ATMs with weak staining (Fig. $3 \mathrm{c}-\mathrm{e}$ ). Moreover, the expression level of IL-1 $\beta$ was positively correlated with the intensity of the pimonidazole signal (Fig. 3f). These results suggest that hypoxia is involved in the inflammatory responses of ATMs in obese adipose tissue.

adipose tissues from 12 week HFD mice were stained with antiCD11c (j, n), anti-F4/80 (k,o), anti-pimonidazole (l,p) or anti-HIF$1 \alpha(\mathbf{m}, \mathbf{q})$ antibody. Representative images are shown. Scale bar is $100 \mu \mathrm{m}$. (r-u) Percentage $(\mathbf{r}, \mathbf{t})$ and number $(\mathbf{s}, \mathbf{u})$ of M1 (black bar) or M2 (white bar) ATMs per total F4/80-positive cells in the epididymal $(\mathbf{r}, \mathbf{s})$ or subcutaneous $(\mathbf{t}, \mathbf{u})$ adipose tissue of chow-fed mice or mice fed an HFD for 4, 8 and 12 weeks. The results are shown as the mean \pm SEM for $4-11$ mice per group. ${ }^{*} p<0.05,{ }^{* *} p<0.01$; WAT, white adipose tissue

Some M1 macrophage markers in SVFs and BMDMs are upregulated by in vitro hypoxia To clarify the causal relationship between hypoxia and the inflammatory polarity of macrophages, we performed in vitro experiments with cultures of SVFs of epididymal adipose tissue (Fig. 4a,b) and BMDMs (Fig. 4c-e). Our flow cytometry analysis showed that approximately $50 \%$ or more of the cells in the SVF were F4/80-positive (data not shown). Cells were cultured in a hypoxic chamber $\left(1 \% \mathrm{O}_{2}\right)$ for $24 \mathrm{~h}$. This in vitro hypoxia treatment significantly upregulated hypoxia-related genes, 

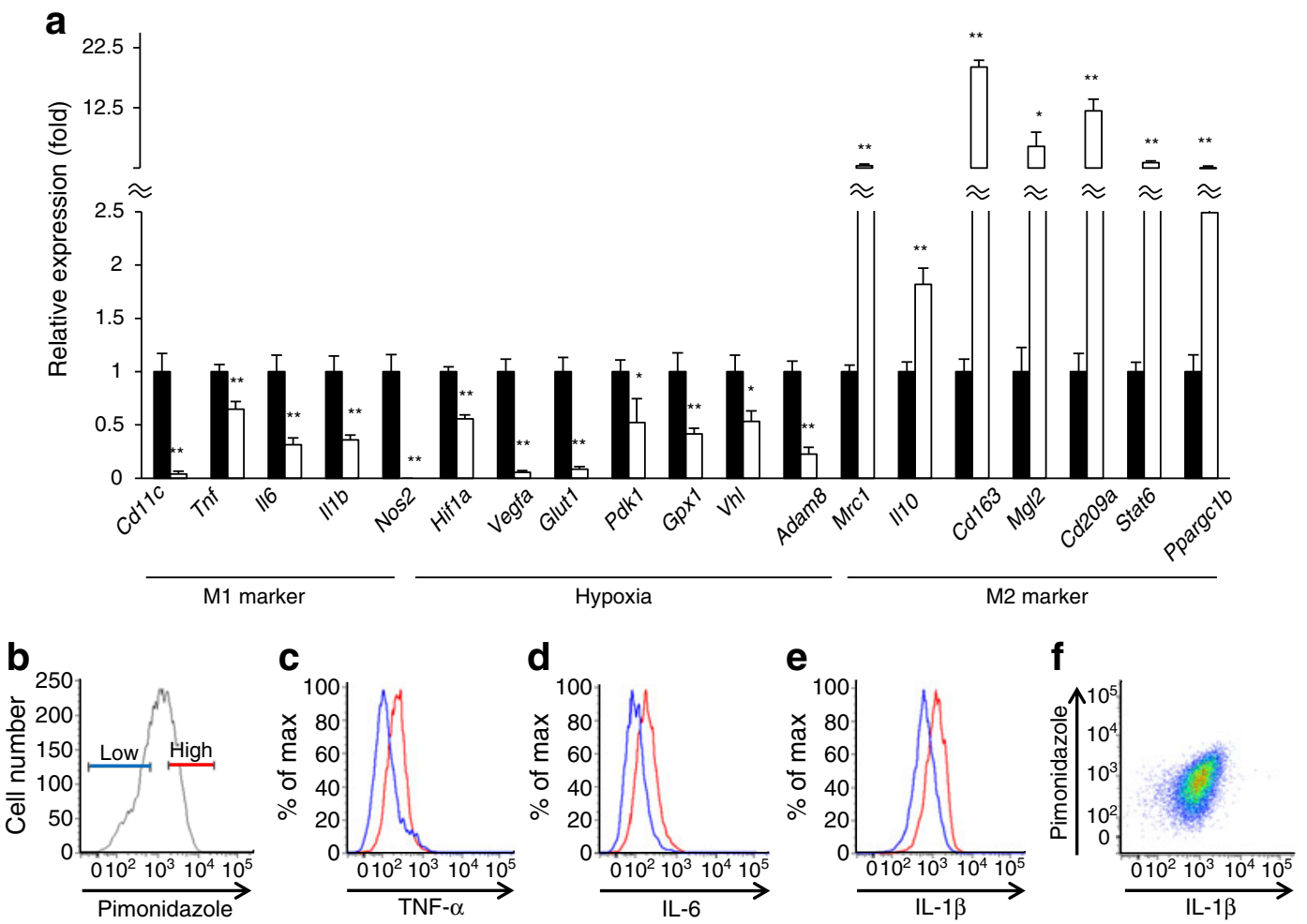

Fig. 3 ATMs expressing hypoxia marker genes produce high levels of inflammatory cytokines. (a) Gene profiles of M1 (black bar) or M2 (white bar) ATMs in high-fat-fed mice. Each data point was normalised according to the $18 \mathrm{~S}$ rRNA level. The results are shown as the mean \pm SEM for five or six mice per group. ${ }^{*} p<0.05,{ }^{* *} p<0.01$. (b-e) Flow cytometry analysis of ATMs from high-fat-fed mice. CD45-positive/ F4/80-positive ATMs were gated and examined according to the levels of pimonidazole staining (b). Thirty per cent of the pimonidazole-high (red line) and $30 \%$ of the pimonidazole-low (blue line) ATMs were further analysed using anti-TNF- $\alpha$ (c), anti-IL-6 (d) or anti-IL-1 $\beta$ (e) antibody. (f) Flow cytometry analysis of ATMs with anti-pimonidazole and anti-IL-1 $\beta$ antibodies. All the data are representative of at least three independent experiments such as Glut1 and Vegfa, with increased HIF-1 $\alpha$ protein in both the SVF and BMDMs. The expression of Cd11c (also known as Itgax), a critical marker for M1 ATMs, and genes for some inflammatory cytokines, including $I l 6, I l l b$ and Nos2, were also significantly upregulated by the exposure to hypoxia (Fig. 4a,c). Interestingly, the expression of Tnf, another representative marker gene for M1 ATM, was significantly downregulated in the SVF (Fig. 4a) and remained unaltered in the BMDMs (Fig. 4c) by the exposure to hypoxia, suggesting that hypoxia does not induce all proinflammatory cytokines. M2 ATM marker genes were not altered in the SVF or downregulated in BMDMs by exposure to hypoxia.

To determine the involvement of HIF-1 $\alpha$ in the induction of M1 marker genes by hypoxia, we next performed similar experiments using BMDMs from $\mathrm{Hifl}^{\text {flox/flox}} / \mathrm{LysM}$-cre mice, in which Hifla was deleted specifically in the monocyte/macrophage compartment (Fig. 4c). More than $90 \%$ deletion of the HIF- $1 \alpha$ protein in the cultured BMDMs was confirmed by western blot (Fig. 4d). The basal expression of the M1 markers, such as Tnf, Il6, Illb and Nos2, was decreased as was the expression of hypoxia- related genes, such as Glut1 and Vegfa, in Hifla-deleted BMDMs. The response to the in vitro hypoxia treatment was attenuated significantly in these genes. Interestingly, the deletion of Hifla increased Cd11c expression under both normoxic and hypoxic conditions (Fig. 4c). Taken together, these results suggest that the expression of most inflammatory M1 markers (excluding $T n f$ ) were, at least in part, induced by hypoxia in an Hifla-dependent manner.

Alternatively activated BMDMs poorly express inflammatory markers during hypoxia Finally, we examined the expression of inflammatory marker genes under hypoxic conditions in M2 macrophages that had been alternatively activated by IL-4 treatment. IL-4 treatment significantly decreased the expression of both inflammation-related genes, such as $I l 6$ and $I l 1 b$, and hypoxia-related genes, such as Glut1 and Vegfa, under normoxic conditions. Interestingly, IL-4 treatment significantly attenuated hypoxia-induced inflammation-related genes, such as $I l 6, I l 1 b$ and Nos2, while it increased hypoxiarelated genes, such as Glut1 and Vegfa (Fig. 4e). These results suggest that the induction of markers of inflammation in 


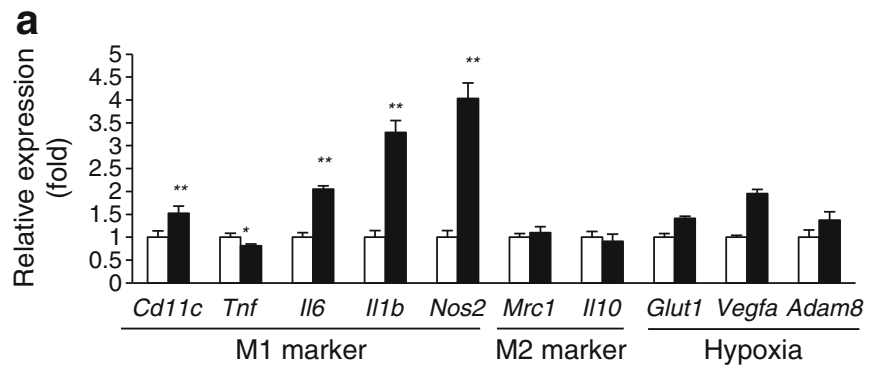

a b

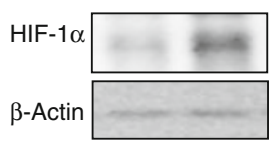

Hypoxia (-) (+)

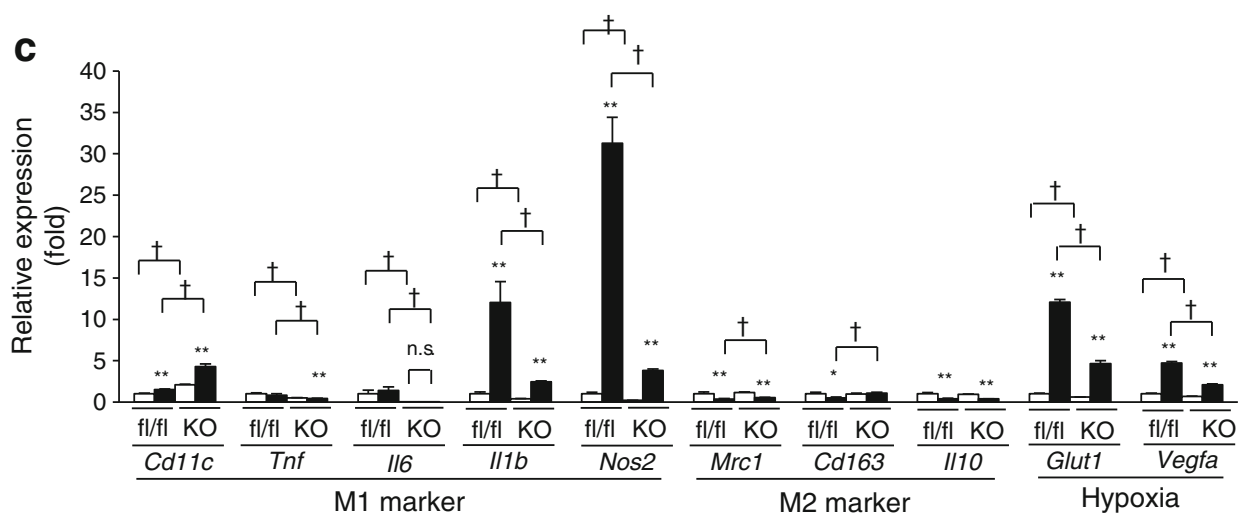

d

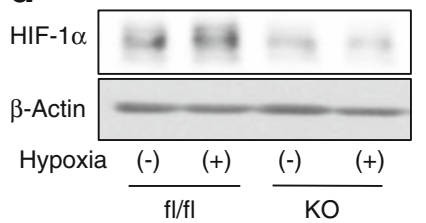

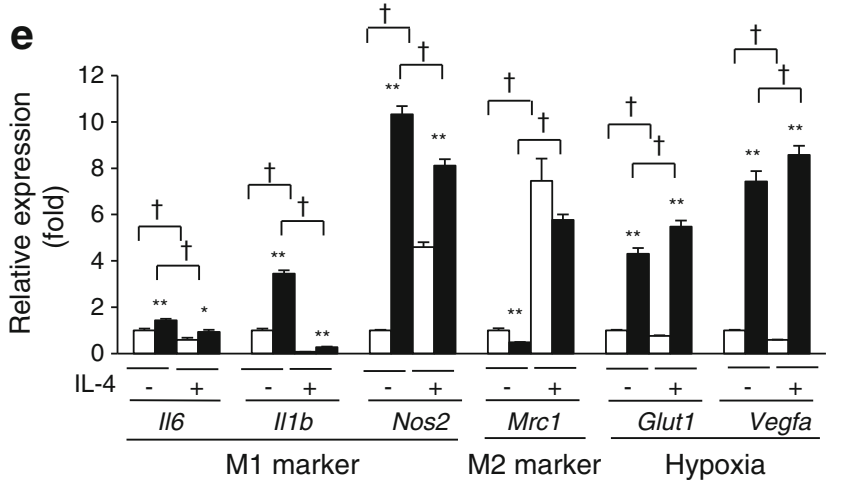

f

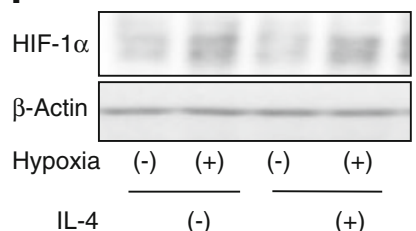

Fig. 4 The expression of M1 macrophage markers is upregulated after in vitro exposure to hypoxia in SVFs and BMDMs. SVF from C57BL/ $6 \mathrm{~J}$ mice $(\mathbf{a}, \mathbf{b}), \mathrm{BMDM}$ from control (fl/fl) or LysM-cre HIF- $1 \alpha$ knockout $(\mathrm{KO})$ mice $(\mathbf{c}, \mathbf{d})$, or BMDM from C57BL/6J mice (e) were treated under conditions of normoxia $\left(20 \% \mathrm{O}_{2}\right.$; white bar) or hypoxia $\left(1 \% \mathrm{O}_{2}\right.$; black bar) for $24 \mathrm{~h}$. BMDM was also treated with or without $10 \mathrm{ng} / \mathrm{ml}$

response to hypoxia is relatively poor in alternatively activated M2 macrophages.

\section{Discussion}

Several recent studies have suggested that hypoxia is involved in the inflammatory changes that occur in obese adipose tissues $[14,15,28]$. However, the direct effects of adipose tissue hypoxia on the inflammatory polarity of ATMs have not been examined. In this study, we were able to demonstrate some novel findings regarding the relationship between adipose tissue hypoxia and ATM polarity. M1 ATMs, which were of IL-4 for $24 \mathrm{~h}$ (e). (a,c,e) Gene expression for each data point was normalised according to the $18 \mathrm{~S}$ rRNA level. The results are shown as the mean \pm SEM for four mice per group. ${ }^{*} p<0.05,{ }^{* *} p<0.01$ vs normoxia; ${ }^{\dagger} p<0.01 \mathrm{vs} \mathrm{fl} / \mathrm{fl}(\mathbf{c})$ or without IL-4 (e). (b,d) Representative western blot images of SVF from C57BL/6J mice (b) or BMDM from fl/fl or KO mice (d) with anti-HIF- $1 \alpha$ or anti- $\beta$-actin antibody

obtained directly from the adipose tissues of obese mice, were more hypoxic than M2 ATMs (Figs 2b-i, 3a, ESM Fig. 1). Macrophages exposed to hypoxia expressed higher levels of M1 marker genes, including those for proinflammatory cytokines, in both in vivo (Fig. 3b-f) and in vitro (Fig. 4) experiments. The induction of most of the M1 marker genes by exposure to hypoxia was dependent on Hifla, at least in part (Fig. 4c). Moreover, we observed that the expression of inflammatory genes in BMDMs that were alternatively activated by in vitro treatment poorly responded to hypoxia (Fig. 4e). These results indicated that adipose tissue hypoxia induces M1-like polarity in ATMs, thus contributing to the inflammatory characteristics of visceral adipose tissue. 
M1 ATMs were more hypoxic than M2 ATMs in vivo (Figs 2, 3 and 4), but the precise mechanisms responsible for the difference between the M1 and M2 ATMs are not fully understood. M1 ATMs mainly exist in CLSs in obese adipose tissue. Cinti et al reported that M1 ATMs are recruited into obese adipose tissue to scavenge lipid droplets from dead adipocytes using their phagocytic activity [29]. The area around a dead adipocyte is often avascular and hypoxic. Damage-associated molecular patterns released from the dead adipocytes might attract M1 macrophages into the avascular hypoxic areas against the oxygen gradient [30]. Since M1 ATMs have to move toward this hypoxic area to clear dead adipocytes, the metabolism of M1 ATMs becomes dependent on anaerobic glycolysis to produce ATP by stabilising HIF- $1 \alpha$ to adapt to the hypoxic environment. As some proinflammatory genes lie downstream of HIF-1 $\alpha$, the M1 ATMs highly expressed not only hypoxia-related metabolic genes, but also inflammatory genes, including $I l 1 b$ and Nos2. Indeed, we found that $P d k 1$, a marker of glycolytic metabolism, was expressed at higher levels in M1 ATMs than in M2 ATMs (Fig. 3a). Furthermore, the anaerobic phenotype is reportedly observed not only in ATMs but also in peritoneal macrophages (i.e. peritoneal macrophages resemble the classically activated M1 macrophages induced by lipopolysaccharide/IFN $\gamma$, the metabolic profiles of which are characterised by enhanced glycolytic activity) [31]. In contrast to M1 ATMs, which are recruited into CLSs, M2 ATMs are evenly distributed in adipose tissues under both obese and non-obese conditions [32]. The different distribution patterns of M1 and M2 ATMs may, at least in part, explain their anaerobic and aerobic metabolism characteristics.

As demonstrated above, hypoxia appears to be a trigger for chronic inflammation in obese adipose tissue. If so, ATMs should experience an inflammatory response following hypoxic changes. However, although the pimonidazole staining in M1 ATMs was stronger than that in M2 ATMs after 8 weeks of high-fat feeding in epididymal adipose tissues (Fig. 2d), the inflammatory genes were already elevated in M1 ATMs after 4 weeks of high-fat feeding (ESM Fig. 1f,g). Pimonidazole reportedly binds to cells or tissues where the oxygen partial pressure is less than $10 \mathrm{mmHg}$ [33]. Thus, we speculated that mild hypoxia occurs before 4 weeks of high-fat feeding and while mild hypoxia cannot be detected by pimonidazole staining, it might be capable of stimulating an inflammatory response in adipose tissues.

Recent studies using a macrophage-specific gene deletion system have demonstrated that the I-kappaB kinase beta $(\mathrm{IKK} \beta)$-nuclear factor-kappaB $(\mathrm{NF}-\mathrm{kB})$ pathway in macrophages is involved in the inflammatory phenotype of obese mice [34]. HIF- $1 \alpha$ is a transcriptional factor that is activated by hypoxic stimuli and can promote inflammatory responses in some cells [35]. However, the involvement of the HIF-1 $\alpha$ pathway in the function of ATMs has not yet been well documented. Thus, it is also important to clarify the relationship between the activation of the HIF-1 $\alpha$ pathway and inflammatory changes in ATMs in vivo. Our present study demonstrated that the HIF- $1 \alpha$ pathway was also involved in the induction of inflammatory changes in ATMs. Of note, in vitro exposure to hypoxia increased the expression of $I l 6$, $I l 1 \mathrm{~b}$ and Nos2, but not that of Tnf in cultured SVFs or BMDMs (Fig. 4). These results suggest that there are at least two pathways leading to the induction of proinflammatory markers in macrophages (i.e. hypoxiadependent and hypoxia-independent pathways). On the other hand, M1 ATMs sorted from adipose tissues expressed not only hypoxia-inducible proinflammatory genes, including $I l 6, I l 1 b$ and Nos2, but also Tnf, (the latter not being induced by in vitro exposure to hypoxia) (Fig. 3a). Similarly, ATMs showing stronger pimonidazole signalling produced more inflammatory cytokines, including TNF- $\alpha$ and IL-1 $\beta$ at the protein level (Fig. 3b-f). The discrepancy in the induction of $\operatorname{Tnf}$ gene expression between the in vivo and in vitro experiments may be explained as follows. In adipose tissue, Tnf in M1 ATMs can be induced not only by hypoxia but also by various other stimuli. For example, inflammatory cytokines produced by other cells can induce Tnf expression in M1 AMTs in a paracrine manner. On the other hand, cultured macrophages are not influenced by the various stimuli after in vitro exposure to hypoxia.

As shown in Fig. 4e, alternatively activated M2 macrophages poorly responded to hypoxic conditions with regard to the expression of inflammation-associated marker genes. A recent study has reported that the activation of NF- $\mathrm{KB}$ plays a role in the accumulation of HIF- $1 \alpha$ protein in cells [36]. If NF-KB activity in M2 macrophages is low, then HIF$1 \alpha$ stability might deteriorate. However, the expression of HIF- $1 \alpha$ target genes, such as Glut 1 and Vegfa, actually increased, suggesting that the transcriptional activity of HIF- $1 \alpha$ was maintained in M2 macrophages. Whether the binding activity of HIF- $1 \alpha$ to the hypoxia responsive element (HRE) site on the promoter lesion of these inflammatory genes deteriorated in M2 macrophages remains to be clarified.

Subcutaneous adipose tissue is reportedly less inflammatory than visceral adipose tissue in obese individuals [23-26]. We showed that most of the ATMs in the subcutaneous adipose tissue in obese mice are M2 (Fig. 2t,u), and the expression of inflammatory markers in alternatively activated BMDMs was relatively poor after hypoxic treatment (Fig. 4e). This poor response of M2 macrophages to hypoxia might explain the less inflammatory characteristics of subcutaneous adipose tissue, to some extent.

Why does adipose tissue become hypoxic during progression of obesity? Some possible mechanisms for these changes have recently been proposed. First, the capillary density is reduced [27] and the blood flow is lower in the 
adipose tissues of obese individuals (30-40\% of the value in non-obese controls) [37]. Consistent with this, we also observed that the expression of vascular endothelial growth factor (VEGF), a well-known growth factor that promotes angiogenesis, was significantly decreased in the adipocyte fraction of obese mice, whereas most of the other hypoxia marker genes were upregulated (Fig. 1c). Second, Elias et al recently reported that adipocyte-specific VEGF overexpression increased the number of blood vessels in adipose tissues, improving high-fat-diet-induced adipose tissue hypoxia and insulin resistance [38]. These results suggest that the insufficient induction of VEGF expression in obesity is one of the causes of adipose tissue hypoxia. The adipose tissue hypoxia observed in obesity may also be associated with the oxygen diffusion distance. In general, oxygen diffuses about $120 \mu \mathrm{m}$ from the tissue capillaries [39, 40], although the adipocyte diameter often becomes $150 \mu \mathrm{m}$ or longer in obese rodents and humans $[41,42]$. In this situation, oxygen cannot reach the opposite side of the large adipocytes from the capillary. Indeed, we found that the expression of hypoxia-related genes was higher in the SVF than in the adipocyte fraction of obese mice (Fig. 1b-j). These results suggest that the SVF is the major site of adipose tissue hypoxia. However, we should not forget that adipocytes are also affected by hypoxia to some extent. Hypoxic adipocytes reportedly produce less adiponectin, exhibit a higher endoplasmic reticulum stress and release larger quantities of NEFA during obesity $[14,15]$. Presumably, we should consider that cross-talk exists between adipocytes and the cells in the SVF and that hypoxia-mediated dysfunction in those cells promotes insulin resistance in a coordinated manner.

In this study, we demonstrated that adipose tissue hypoxia induces inflammatory M1 activation of ATMs via HIF$1 \alpha$-dependent and HIF- $1 \alpha$-independent mechanisms. This process, from adipose tissue hypoxia to inflammatory activation, is expected to become a new therapeutic target for obesity-associated metabolic diseases.

Acknowledgements We thank M. Nangaku (Tokyo University) and Y. Koshimizu (University of Toyama) for helpful discussion and K. Sugihara, Z. Qun, H. Hatta and T. Kumada (University of Toyama) for technical assistance.

Funding This work was supported by Grants-in-Aid for Scientific Research from the Ministry of Education, Science, Sports, and Culture, Japan (18209033 and 21591126 to K. Tobe, 22590971 to I. Usui and 30512082 to S. Fujisaka), by Grant-in-Aid for Scientific Research from Japan Society for the Promotion of Science (JSPS) (24390119 for M. Ikutani, Y. Nagai, K. Takatsu) and by MEXT-Supported Program for the Strategic Research Foundation at Private Universities for N. Goda.

Duality of interest The authors declare that there is no duality of interest associated with this manuscript.

Contribution statement SF acquired and analysed the data, and drafted the article. IU designed the project, interpreted the data and drafted the article. MI acquired and analysed the data (flow cytometry and cell sorting) and revised the article. AA and AT acquired the data (western blot) and revised the article. KTs acquired the data (immunohistochemistry) and revised the article. AM and NG analysed and interpreted the data (experiments using knockout mice) and revised the article. YN and KTa analysed and interpreted the data (flow cytometry and cell sorting) and revised the article. KTo designed the project, interpreted the data and drafted the article. All authors approved the final version of the paper to be published.

\section{References}

1. Hotamisligil GS (2006) Inflammation and metabolic disorders. Nature 444:860-867

2. Weisberg SP, McCann D, Desai M, Rosenbaum M, Leibel RL, Ferrante AW Jr (2003) Obesity is associated with macrophage accumulation in adipose tissue. J Clin Investig 112:1796-1808

3. Xu H, Barnes GT, Yang Q et al (2003) Chronic inflammation in fat plays a crucial role in the development of obesity-related insulin resistance. J Clin Investig 112:1821-1830

4. Virtue S, Vidal-Puig A (2010) Adipose tissue expandability, lipotoxicity and the metabolic syndrome - an allostatic perspective. Biochim Biophys Acta 1801:338-349

5. Gordon S, Taylor PR (2005) Monocyte and macrophage heterogeneity. Nat Rev Immunol 5:953-964

6. Mantovani A, Sica A, Sozzani S, Allavena P, Vecchi A, Locati M (2004) The chemokine system in diverse forms of macrophage activation and polarization. Trends Immunol 25:677-686

7. Fujisaka S, Usui I, Bukhari A et al (2009) Regulatory mechanisms for adipose tissue M1 and M2 macrophages in diet-induced obese mice. Diabetes 58:2574-2582

8. Fujisaka S, Usui I, Kanatani Y et al (2011) Telmisartan improves insulin resistance and modulates adipose tissue macrophage polarization in high-fat-fed mice. Endocrinology 152:1789-1799

9. Charo IF (2007) Macrophage polarization and insulin resistance: PPARgamma in control. Cell Metabol 6:96-98

10. Odegaard JI, Ricardo-Gonzalez RR, Goforth MH et al (2007) Macrophage-specific PPARgamma controls alternative activation and improves insulin resistance. Nature 447:1116-1120

11. Bouhlel MA, Derudas B, Rigamonti E et al (2007) PPARgamma activation primes human monocytes into alternative M2 macrophages with anti-inflammatory properties. Cell Metabol 6:137-143

12. Kang K, Reilly SM, Karabacak V et al (2008) Adipocyte-derived Th2 cytokines and myeloid PPARdelta regulate macrophage polarization and insulin sensitivity. Cell Metabol 7:485-495

13. Sun K, Kusminski CM, Scherer PE (2011) Adipose tissue remodeling and obesity. J Clin Investig 121:2094-2101

14. Ye J, Gao Z, Yin J, He Q (2007) Hypoxia is a potential risk factor for chronic inflammation and adiponectin reduction in adipose tissue of ob/ob and dietary obese mice. Am J Physiol Endocrinol Metab 293:E1118-E1128

15. Hosogai N, Fukuhara A, Oshima K et al (2007) Adipose tissue hypoxia in obesity and its impact on adipocytokine dysregulation. Diabetes 56:901-911

16. Rausch ME, Weisberg S, Vardhana P, Tortoriello DV (2008) Obesity in $\mathrm{C} 57 \mathrm{BL} / 6 \mathrm{~J}$ mice is characterized by adipose tissue hypoxia and cytotoxic T cell infiltration. Int J Obes (Lond) 32:451-463

17. Wang B, Wood IS, Trayhurn P (2007) Dysregulation of the expression and secretion of inflammation-related adipokines by hypoxia in human adipocytes. Pflugers Arch 455:479-492

18. Larsen OA, Lassen NA, Quaade F (1966) Blood flow through human adipose tissue determined with radioactive xenon. Acta Physiol Scand 66:337-345 
19. Kabon B, Nagele A, Reddy D et al (2004) Obesity decreases perioperative tissue oxygenation. Anesthesiology 100:274-280

20. Nishimura S, Manabe I, Nagasaki M et al (2007) Adipogenesis in obesity requires close interplay between differentiating adipocytes, stromal cells, and blood vessels. Diabetes 56:1517-1526

21. Halberg N, Khan T, Trujillo ME et al (2009) Hypoxia-inducible factor 1alpha induces fibrosis and insulin resistance in white adipose tissue. Mol Cell Biol 29:4467-4483

22. Divoux A, Tordjman J, Lacasa D et al (2010) Fibrosis in human adipose tissue: composition, distribution, and link with lipid metabolism and fat mass loss. Diabetes 59:2817-2825

23. Fried SK, Bunkin DA, Greenberg AS (1998) Omental and subcutaneous adipose tissues of obese subjects release interleukin-6: depot difference and regulation by glucocorticoid. J Clin Endocrinol Metab 83:847-850

24. Bruun JM, Lihn AS, Madan AK et al (2004) Higher production of IL-8 in visceral vs. subcutaneous adipose tissue. Implication of nonadipose cells in adipose tissue. Am J Physiol Endocrinol Metab 286:E8-E13

25. Li H, Lelliott C, Hakansson P et al (2008) Intestinal, adipose, and liver inflammation in diet-induced obese mice. Metab Clin Exp 57:1704-1710

26. O'Rourke RW, White AE, Metcalf MD et al (2012) Systemic inflammation and insulin sensitivity in obese IFN-gamma knockout mice. Metab Clin Exp 61:1152-1161

27. Pasarica M, Sereda OR, Redman LM et al (2009) Reduced adipose tissue oxygenation in human obesity: evidence for rarefaction, macrophage chemotaxis, and inflammation without an angiogenic response. Diabetes 58:718-725

28. Yin J, Gao Z, He Q, Zhou D, Guo Z, Ye J (2009) Role of hypoxia in obesity-induced disorders of glucose and lipid metabolism in adipose tissue. Am J Physiol Endocrinol Metab 296:E333-E342

29. Cinti S, Mitchell G, Barbatelli G et al (2005) Adipocyte death defines macrophage localization and function in adipose tissue of obese mice and humans. J Lipid Res 46:2347-2355

30. Miyake Y, Yamasaki S (2012) Sensing necrotic cells. Adv Exp Med Biol 738:144-152
31. Rodriguez-Prados JC, Traves PG, Cuenca J et al (2010) Substrate fate in activated macrophages: a comparison between innate, classic, and alternative activation. J Immunol 185:605-614

32. Lumeng CN, DelProposto JB, Westcott DJ, Saltiel AR (2008) Phenotypic switching of adipose tissue macrophages with obesity is generated by spatiotemporal differences in macrophage subtypes. Diabetes 57:3239-3246

33. Gross MW, Karbach U, Groebe K, Franko AJ, Mueller-Klieser W (1995) Calibration of misonidazole labeling by simultaneous measurement of oxygen tension and labeling density in multicellular spheroids. Int J Cancer 61:567-573

34. Arkan MC, Hevener AL, Greten FR et al (2005) IKK-beta links inflammation to obesity-induced insulin resistance. Nat Med 11:191-198

35. Eltzschig HK, Carmeliet P (2011) Hypoxia and inflammation. N Engl J Med 364:656-665

36. Rius J, Guma M, Schachtrup C et al (2008) NF-kappaB links innate immunity to the hypoxic response through transcriptional regulation of HIF-1alpha. Nature 453:807-811

37. Bolinder J, Kerckhoffs DA, Moberg E, Hagstrom-Toft E, Arner P (2000) Rates of skeletal muscle and adipose tissue glycerol release in nonobese and obese subjects. Diabetes 49:797-802

38. Elias I, Franckhauser S, Ferre T et al (2012) Adipose tissue overexpression of vascular endothelial growth factor protects against diet-induced obesity and insulin resistance. Diabetes 61:1801-1813

39. Helmlinger G, Yuan F, Dellian M, Jain RK (1997) Interstitial pH and $\mathrm{pO} 2$ gradients in solid tumors in vivo: high-resolution measurements reveal a lack of correlation. Nat Med 3:177-182

40. Ye J (2011) Adipose tissue vascularization: its role in chronic inflammation. Curr Diabetes Rep 11:203-210

41. Kursawe R, Eszlinger M, Narayan D et al (2010) Cellularity and adipogenic profile of the abdominal subcutaneous adipose tissue from obese adolescents: association with insulin resistance and hepatic steatosis. Diabetes 59:2288-2296

42. Matsubara Y, Kano K, Kondo D, Mugishima H, Matsumoto T (2009) Differences in adipocytokines and fatty acid composition between two adipocyte fractions of small and large cells in high-fat diet-induced obese mice. Ann Nutr Metab 54:258-267 